\title{
Research Paper \\ Assessing and Comparing of Balance and Flexibility Among Elderly Men and Women in the Age Group of 60-79 Years
}

\author{
*Vahid Valipour Dehnou ${ }^{1} \odot$, Reza Motamedi² \\ 1. Department of Physical Education, Faculty of Literature and Humanities, Lorestan University, Khorramabad, Iran. \\ 2. Department of Physical Education, Borujerd Branch, Islamic Azad University, Borujerd, Iran.
}

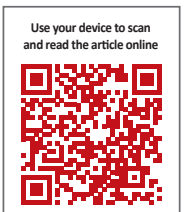

Chtation: Valipour Dehnou V, Motamedi R. [Assessing and Comparing of Balance and Flexibility Among Elderly Men and Women in the Age Group of 60-79 Years (Persian)]. Iranian Journal of Ageing. 2018; 13(2):210-221. https://doi.org/10.32598/sija.13.2.210

Received: 11 Oct 2017 Accepted: 23 Mar 2018

Key words: Physical fitness, Flexibility, Balance, Elderly females, Elderly males

\begin{abstract}
A B S T R A C T
Objectives Functional fitness is a concept that reflects an older adult's ability to perform physical activities of daily living with relative ease. The aim of the present study is to assess and compare balance and flexibility in elderly men and women in the age group of 60-79 years in Khorramabad.

Methods \& Materials In this descriptive and comparative study, 140 elderly people (35 males and 35 females aged 60 to 69 years and 35 males and 35 females aged 70 to 79 years) were conveniently selected and voluntarily participated. To examine balance and flexibility, functional reach and sit and reach tests were used, respectively. For ease of use, tests were performed at the subjects' residence. Independent samples t-test was used to identify any significant difference, and statistical significance was set at $p<0.05$. Results Results showed that significant differences were observed between balance and flexibility of males aged 60 to 69 years and 70 to 79 years $(P<0.05)$; significant differences were observed between balance and flexibility of females aged 60 to 69 years and 70 to 79 years $(P<0.05)$; significant differences were observed between balance and flexibility of males and females aged 60 to 69 years and 70 to 79 years $(P<0.05)$ and 69 to 79 years. Balance and flexibility of both males and females decreased progressively, and the rate of decrement was greater in females than males (-15.79\% vs. $-14.55 \%$ for balance and $-17.79 \%$ vs. $-12.63 \%$ for flexibility).

Conclusion Given the significant role of balance and flexibility in the better performance of daily activities and the reducing them in elderly men and women, it is important to reinforce these two factors of physical fitness throughout life. And to reinforce them, a multicomponent exercise intervention could be the best option.
\end{abstract}

\section{Extended Abstract}

\section{Objectives}

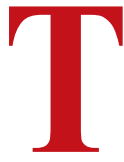

oday, due to the rising life expectancy, aging has become a global epidemic as it is associated with deterioration of ability of individuals to live independently and deterioration in the quality of good life [1]. In addition, due to the advances in health and medicine over the past century, generally, life expectancy and the population of elderly people has increased [2]. Aging is generally associated with a progressive decline in physical activity, and the concept of functional readiness reflects the elderly's ability to carry out the physical activities of everyday life with relative ease. It is proven that age-related functional declines include muscle strength, flexibility, balance, agility, jogging, and cardio-respiratory

\section{* Corresponding Author:}

Vahid Valipour Dehnou, PhD

Address: Department of Physical Education, Faculty of Literature and Humanities, Lorestan University, Khorramabad, Iran.

Tel: +98 (916) 6691874

E-mail: valipour.v@lu.ac.ir 

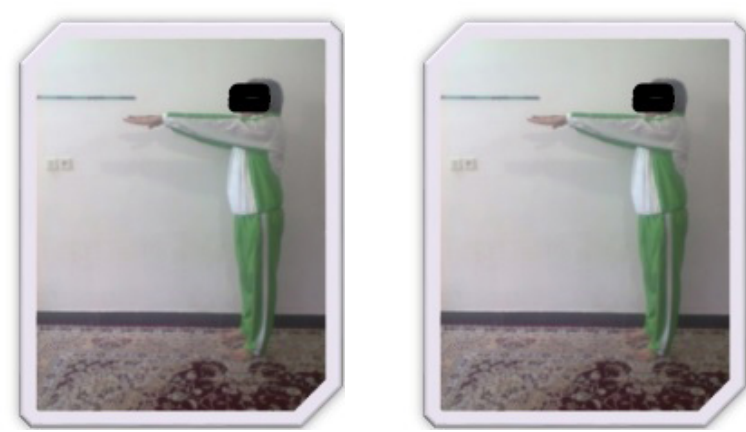

Figure 1. Balance Test (functional each)

ARGEING

readiness, that have a negatively impact on the quality of life [2-4]. Therefore, the purpose of this study was to evaluate and compare the balance and flexibility of elderly women and men aged 60 to 79 years in Khorramabad city and provide solutions to strengthen them.

\section{Methods and Materials}

This cross-sectional descriptive-comparative study was conducted in 2014 in Khorramabad city and the study population consisted of elderly people in the age group of 60 to 79 years. A total of 140 elderly people were selected as a statistical sample with available sampling methods and volunteers for research. Subjects were divided into four groups (35 men and 35 women aged 60-69 years, and 35 men and 35 women in the age group of 70 to 79 years) and were examined. Inclusion criteria were: should be in the age group of 60 to 79 years, inclination to participate in research, lack of physicalmotor disability, not suffering from cognitive or psychological impairment, according to elderly family members, not history of fracture in the recent past and no illness that would

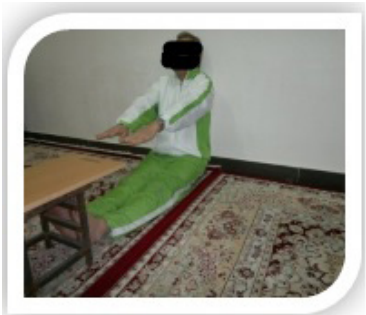

Figure 2. Sit and Reach Test

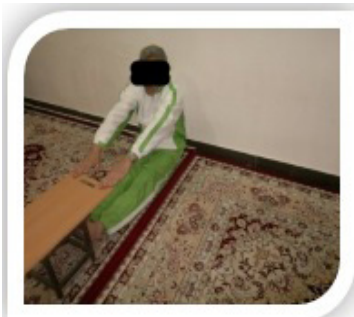

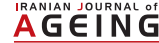

prevent them from taking the tests properly. The exclusion criteria were error during the tests and not having enough ability to do tests. First, the desired test was administered by the researcher, after that, each of the subjects performed it experimentally. After performing the tests properly, the subject performed specific stretching exercises and then the test was administered. Each subject performed 3 special tests and their average was recorded as the subject score. Balance and flexibility tests (Figures 1 and 2) were administered between 16:00 and 18:00 hrs for all the subjects. To assess the balance status, stretching the hands forward in the standing position (functional reach) (dynamic equilibrium) and [3, 5] and to evaluate flexibility, the sit and pass test were used $[6,7]$. The method of this study was approved by the Ethics Committee of Islamic Azad University, Boroujerd Branch. Independent t-test was used to examine the differences and the percentage change was used to check the amount of reduction in the age group of 69-60 years to 70-79 years.

\section{Results}

In this study, 140 elderly men and women, residents of Khorramabad city, were investigated including 70 men and 70 women. Descriptive statistics showed that balance

Table 1. Independent and independent t-tests

\begin{tabular}{cccc}
\hline Indices & Groups & T-Test & P \\
Variables & Women aged 60-69/aged 70-79 & 3.621 \\
\hline Balance & Men aged 60-69/aged 70-79 & 0.001 & 4.961 \\
& Women aged 60-69/aged 70-79 & 0.001 & 3.324 \\
& Men aged 60-69/aged 70-79 & 0.004 & 3.554 \\
\hline Flexibility & Women aged 60-69/aged 70-79 & 0.003 & 3.772 \\
& Men aged 60-69/aged 70-79 & 0.001 & 3.506 \\
\hline & Women aged 60-69/aged 70-79 & 0.001 & 2.984 \\
\hline
\end{tabular}


in women and men in the age group of 69-60 years was $20.90 \pm 3.09$ and $24.60 \pm 4.08 \mathrm{~cm}$, respectively, and it was $17.67 \pm 4.40$ and $21.02 \pm 3.70 \mathrm{~cm}$ in the age group of 70-79 years, respectively. The findings also showed that flexibility in men and women aged 69-60 years was $26.03 \pm 2.80$ and $28.50 \pm 3.80 \mathrm{~cm}$, respectively, and in the age group of 70-79 years, it was $21.4 \pm 5.60$ and $24.90 \pm 3.70 \mathrm{~cm}$, respectively. These findings clearly show that in every age range, men's balance and flexibility are more than women. Inferential statistics are presented in Table 1.

These results showed that there was a significant difference between the balance and flexibility of women and men in the age group of 60-69 years and 70-79 years $(\mathrm{P}<0.05)$. There was a significant difference between the balance and flexibility of men aged $60-69$ years and $70-79$ years $(\mathrm{P}<0.05)$. There was also a significant difference between the balance and flexibility of women aged 60-69 and 70-79 years $(\mathrm{P}<0.05)$. Balance and flexibility in both men and women showed a progressive decline from the age of 60 to 79 , and this decline was higher in women than in men. To calculate the percentage change of the mean scores in each age range, the following formula was used:

(Post Test - Pre Test / Pre Test) $\times 100$

The percentage change is as follows: -15.79 versus -14.55 percent for balance and -17.79 versus $-12.63 \%$ for flexibility. This difference in the balance between men and women may be justified with the amount of daily physical activity and the type of physical activity of men and women in the society. The results of the balance show that men's balance is better than women in every age range and the difference in the strength of the lower extremity muscles perhaps may be able to justify well the difference between the two genders as well as between two age groups in men and women. Given the gender differences, most studies showed that women have more flexibility than men of all ages [8]. The reason for the difference of the results of this study may be due to the daily activities of the subjects in the study, who actively participated in daily life activities, and also men are naturally more active than women.

\section{Conclusion}

Inevitable reduction in the skeletal muscle mass and its associated decreased strength with age [9-11] can justify lower balance and flexibility of individuals in the age group of 79-70 years, compared to those in the age group of 69-60 years. In this regard, Roma et al. showed that two days a week of strength training for six months or a year increased the elderly's balance and flexibility [12]. Therefore, physical activity is recommended for the elderly. Weight-bearing ex- ercises can mainly increase balance and flexibility. Strength exercise is also enhanced by improving strength, ability, mechanics and speed walking and helps in improving balance and thus reduces falls. Resistance stretching can increase flexibility. Considering the decline of the faster pace of balance and flexibility in women, they need to pay more attention to their balance and flexibility as compared to men.

\section{Ethical Considerations}

\section{Compliance with ethical guidelines}

The ethics committee of Islamic Azad University of Boroujerd Branch has approved this study.

\section{Funding}

This research did not receive any specific grant from funding agencies in the public, commercial, or not-forprofit sectors.

\section{Conflict of interest}

The authors declare no conflict of interest.

\section{Acknowledgements}

We thank all the subjects of this study because of their participation in the study and the excellent cooperation with the researcher. 


\title{
بررسى و مقايسةُ تعادل و انعطافيذيرى مردان و زنان سالمند در دامنه سنى +7 تا V9 سال
}

\author{
"وحيد ولىيور ده نو' م. رضا معتمدى'

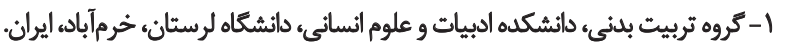

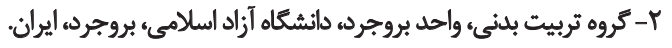

\begin{abstract}
حكSد

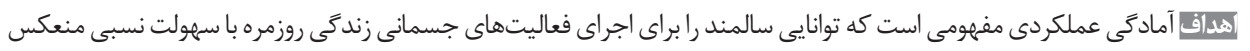

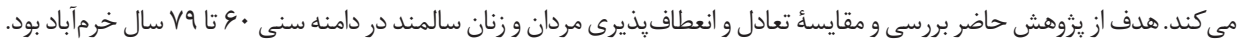

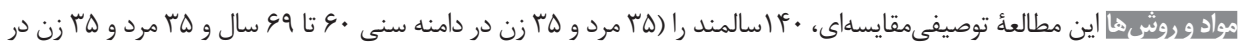

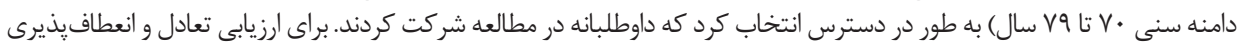

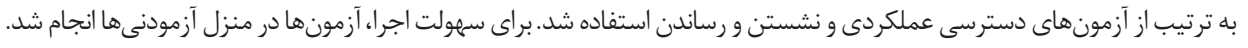

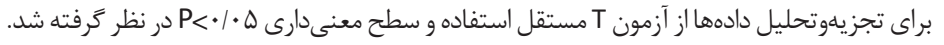

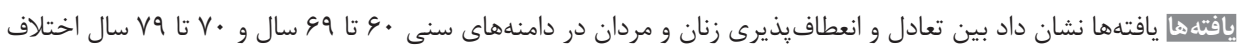

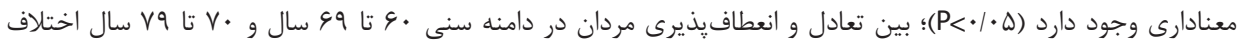

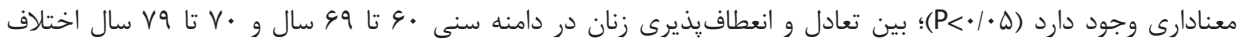

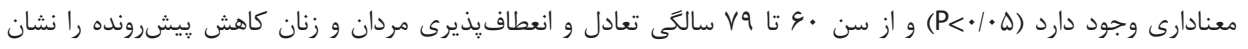

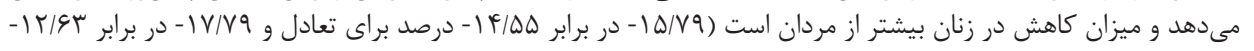
درصد براى انعطافٍ يذيرى).

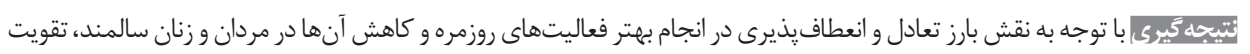

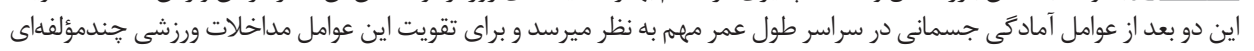
مى تواند بهترين كز ينه باشد.
\end{abstract}

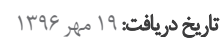

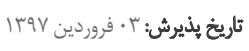

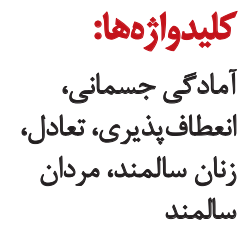

مىشود [F].

مقدمه

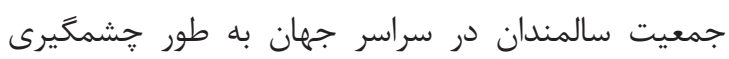

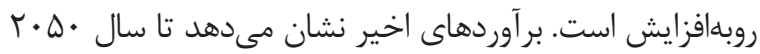

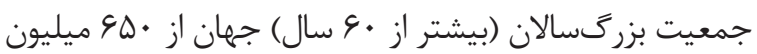

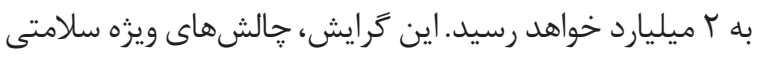

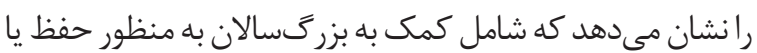

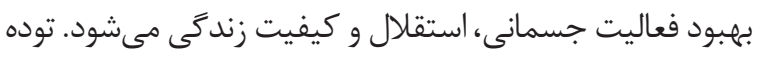

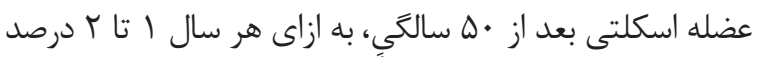

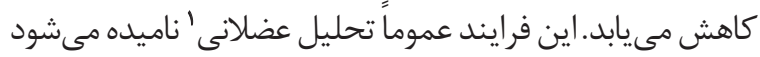

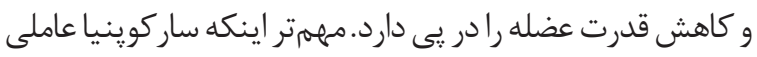
كليدى در كمك به سستى، كاهش تحرى دارد و واستقلال عملكردى،

1. Sarcopenia

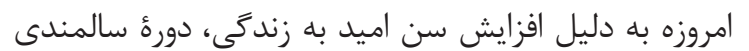

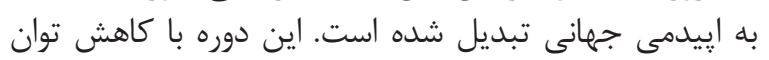

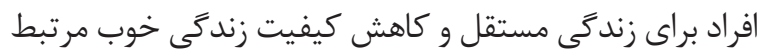

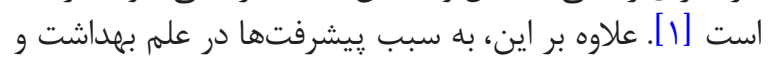

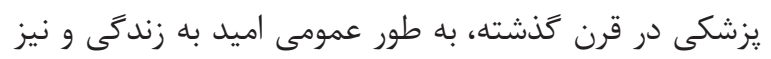

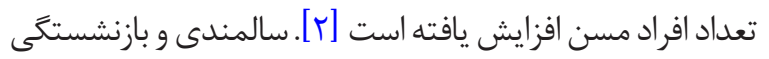

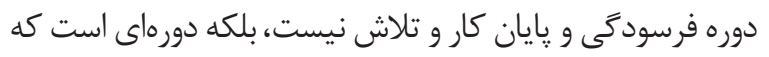

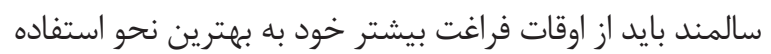

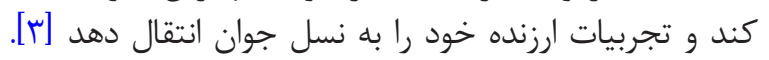

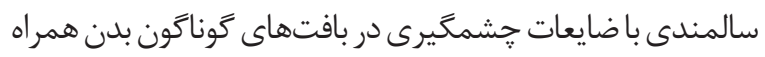

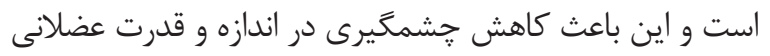

: نويسينده مسينول:

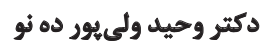

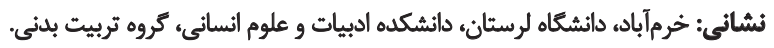

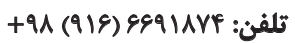
valipour.v@lu.ac.ir يست الكترونيكي: 
حركتى آن است و براى اجراى ورزشى و توانايى انجام فعاليتهاى

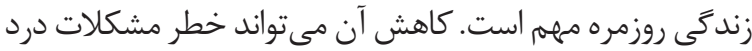

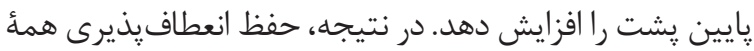

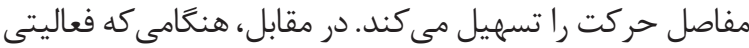

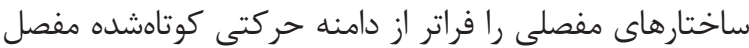

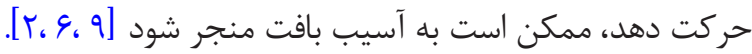

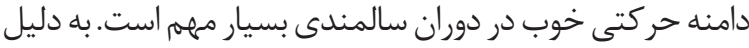

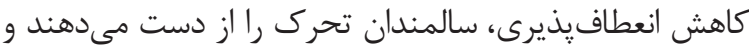

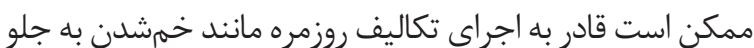

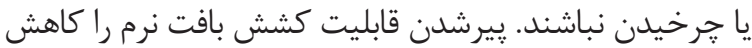

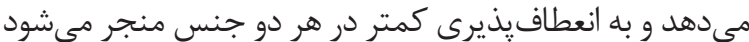

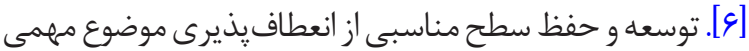

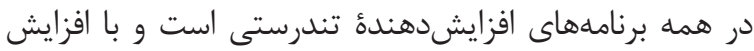

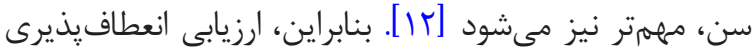

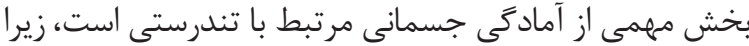

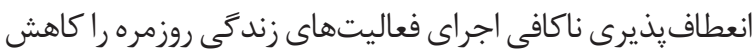

مى مدهد [9].

بيشتر مطالعات نشان دادهاند انعطافٍ يذيرى يا كاهش دامنه

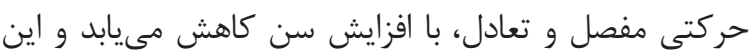

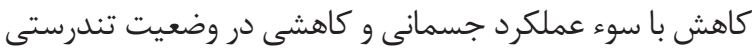
مرتبط است. همجنين انعطافيذيرى زنان إنان در مقايسه با با مردان

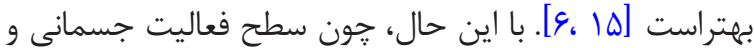

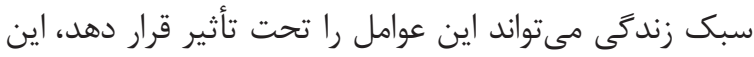

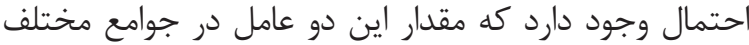

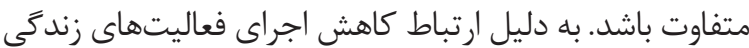

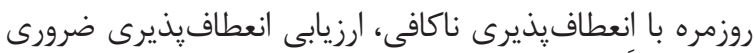

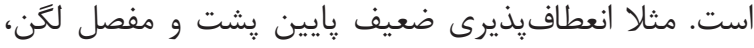

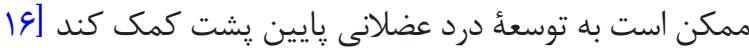

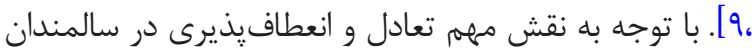

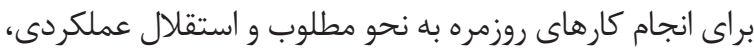

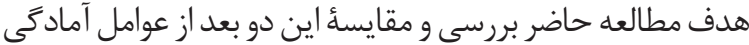

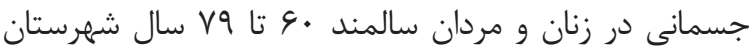
خرمآباد و ارائه راهكارهاى مناسب بردان تقان تقويت آنهاست.

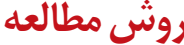

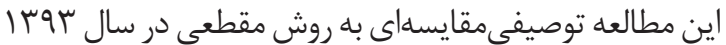

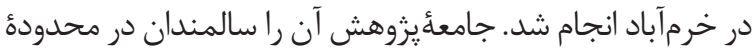

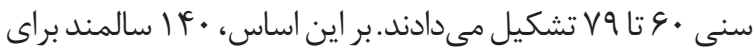

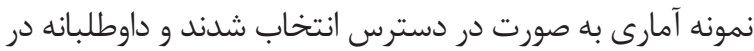

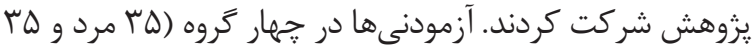

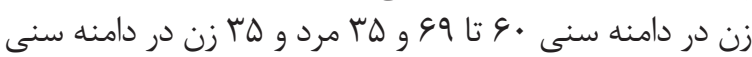

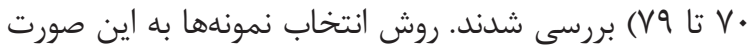

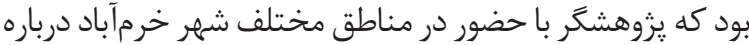

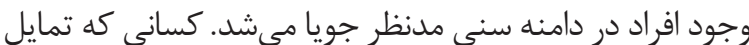

كاهش كيفيت زندگى، كاهش ثبات، افزايش خطر افتادن و

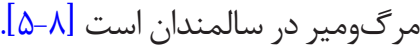

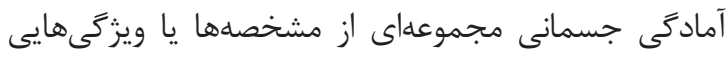

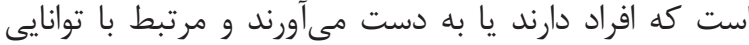

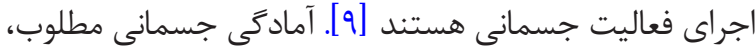

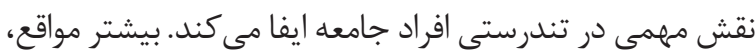

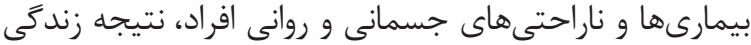

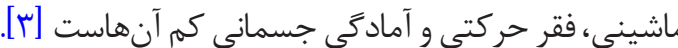
افزايش سن، عموماً با كاهشى ييشرونده در فعاليت جسمانى

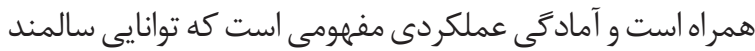

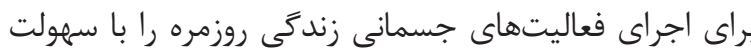

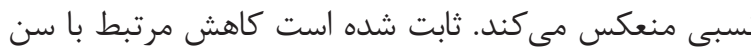

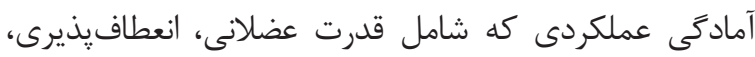

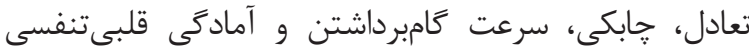

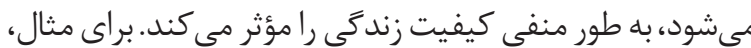

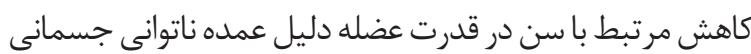

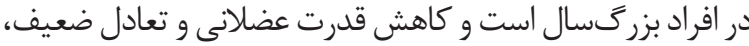

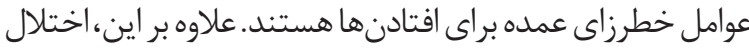

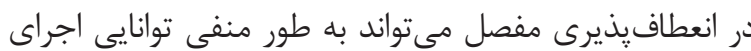

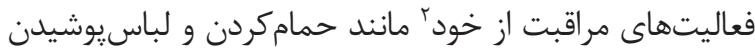

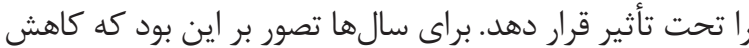

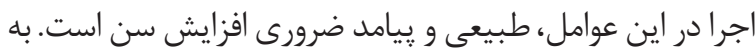

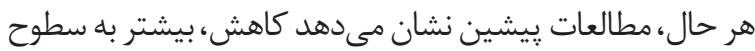

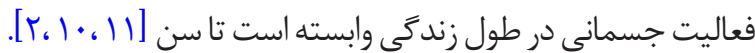
تعادل، فرايند حفظ وضعيت مركز ثقل بدن به طور عمودى

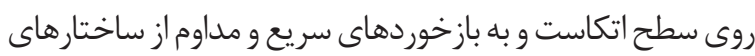

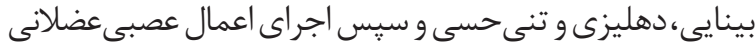

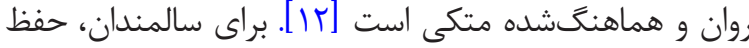

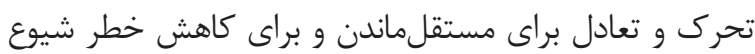

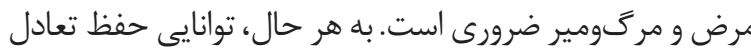

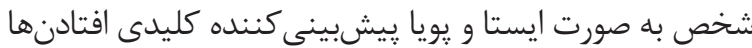

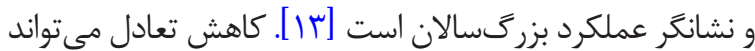

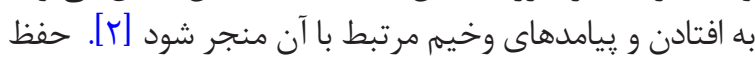

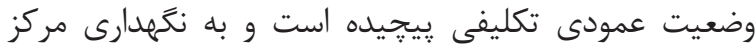

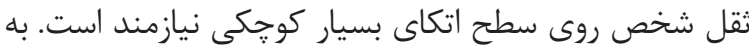

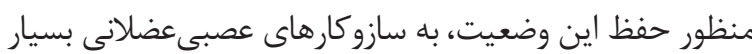

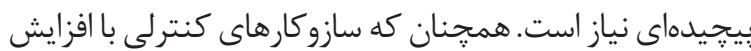

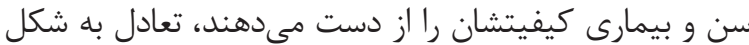

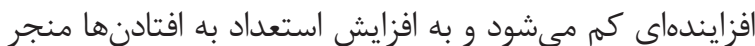

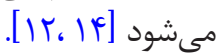

انعطافيذيرى، توانايى حركت مفصل در سراسر دامنهُ كامل

\section{Self-care}




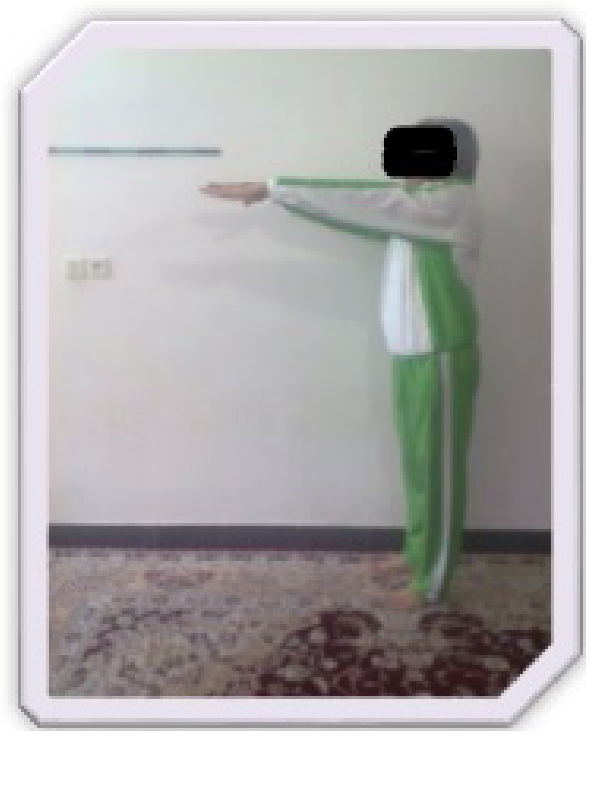

وضعيت ايستاده (دسترسى عملكردى") (تعادل يويا) استفاده شد.

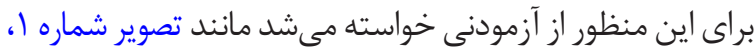

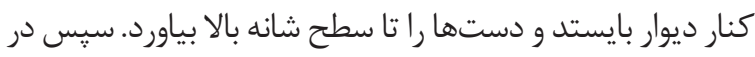

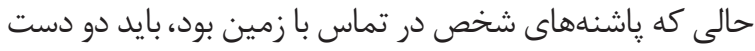

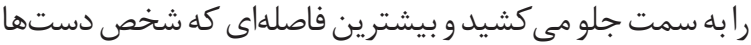

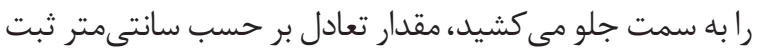

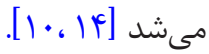

انعطاف يذيرى

براى ارزيابى انعطافيذيرى، از آزمون نشستن و رساندن

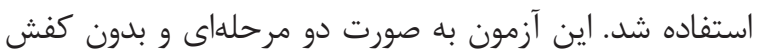

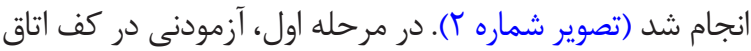

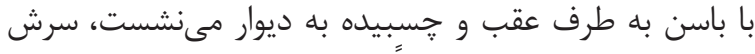

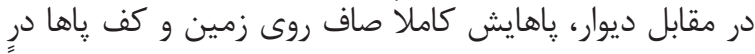

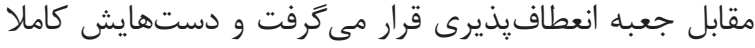

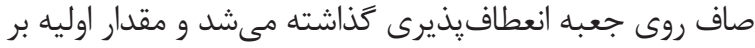

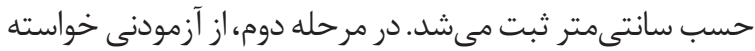

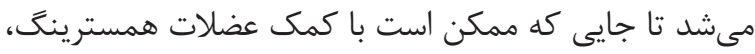

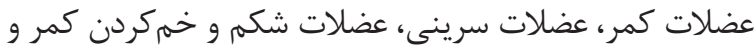

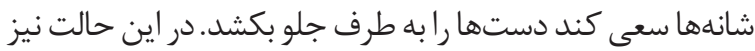

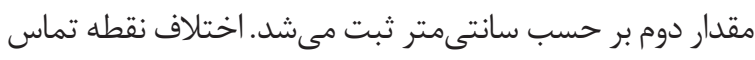

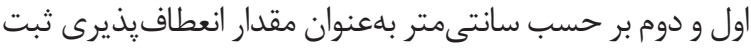

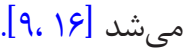
نتايج آزمون كلموتروف-اسميرنف نشان داد دادهها توزيع

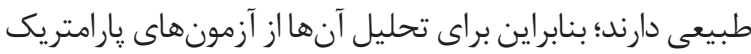

3. Functional reach

4. Sit and reach test

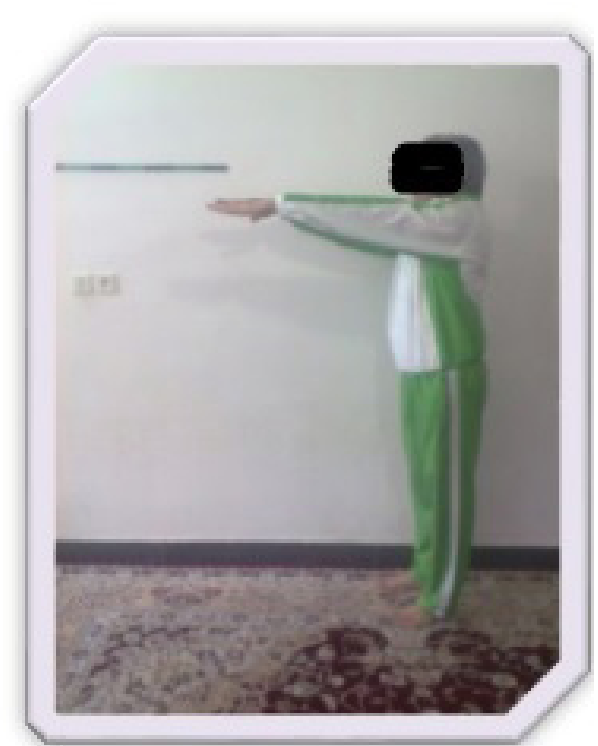

تصوير ا.ـزآمون تعادل (دسترسى عملكردى)

به انجام آزمون داشتند، ثبتنام مى شدند و از آنها آزمونهاى

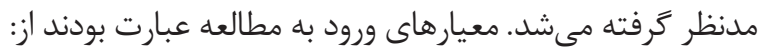

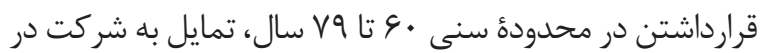

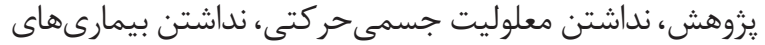

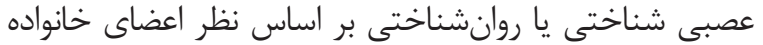

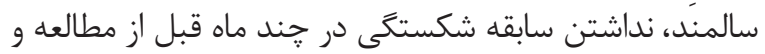

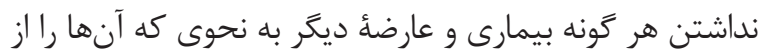

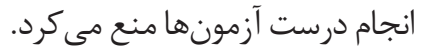

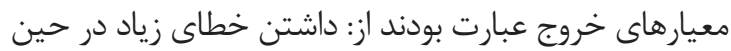

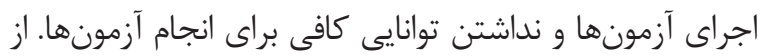

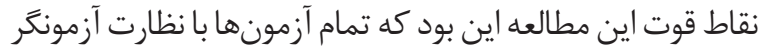

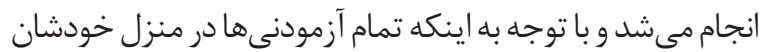

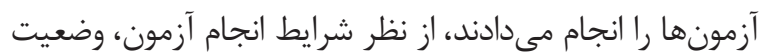

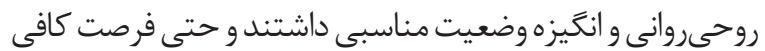
براى انجام آزمونها را داشتند. كميته اخلاق دانشكاه آزاد اسلامى إنى إنى

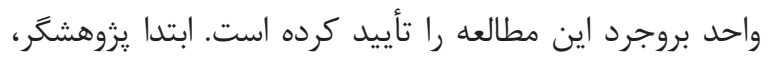

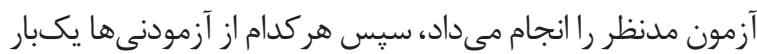

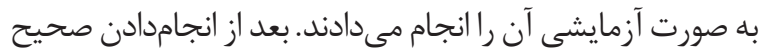

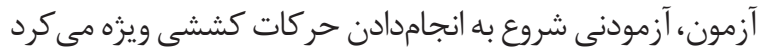

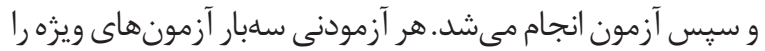

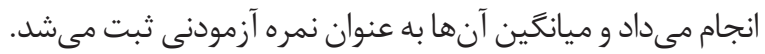

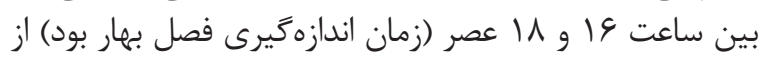

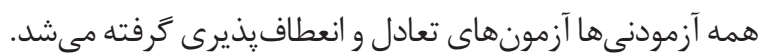

تعادل براى ارزيابى تعادل، از آزمون رساندن دستها به سمت جلو در 


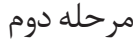

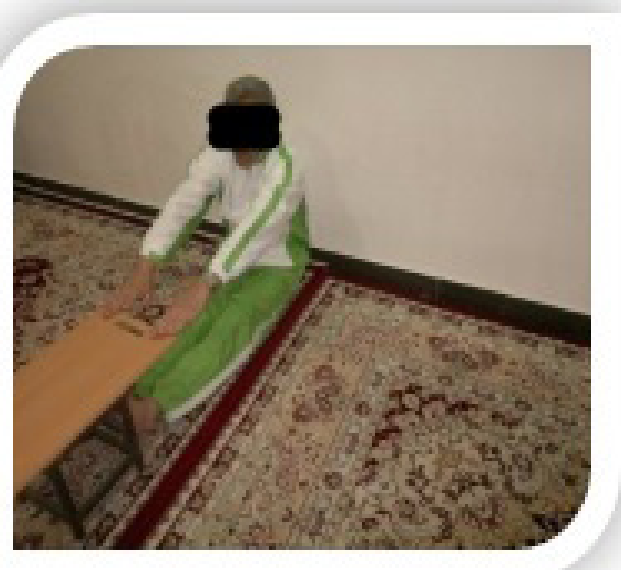

L

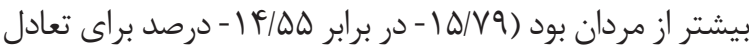

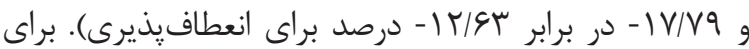

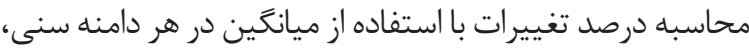

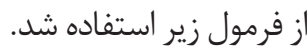

\section{(Post Test - Pre Test/Pre Test) $\times 100$}

\section{بحث}

هدف از يزوهش حاضر بررسى و مقايسة تعادل و انعطافيذيرى

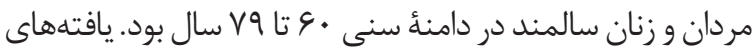

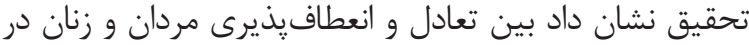

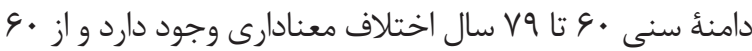

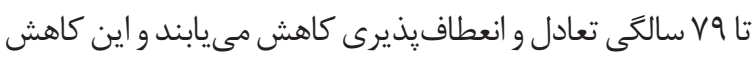

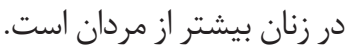

بهخوبى نشان داده شده است فرايند افزايش سن انسان، از بلوغ

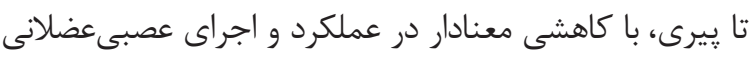

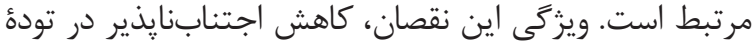

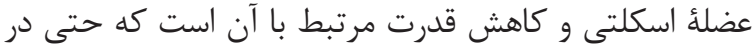

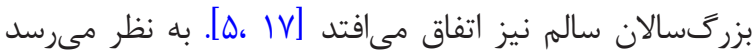

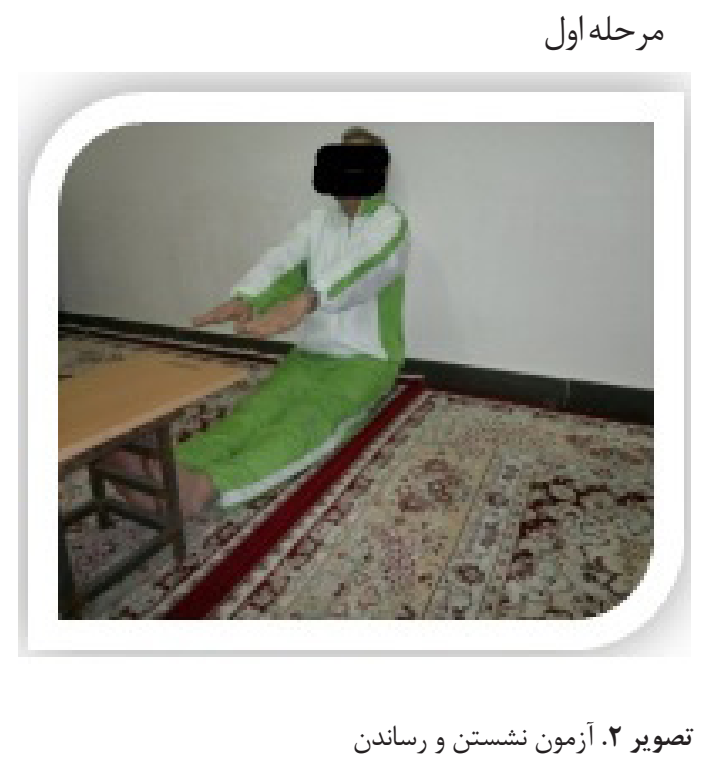

استفاده شد. براى بررسى اختلاف احتمالى بين دو گروه زنان و و و

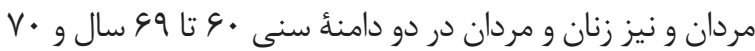

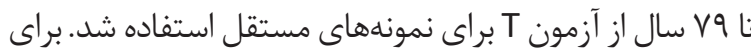

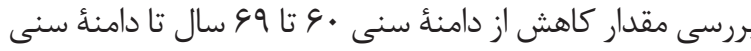

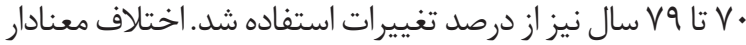

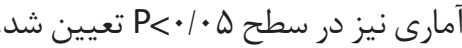

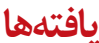

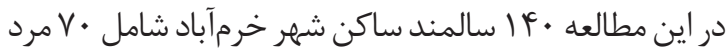

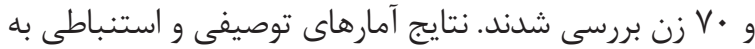

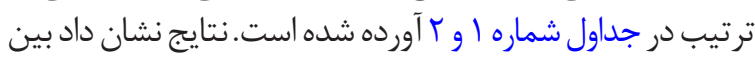

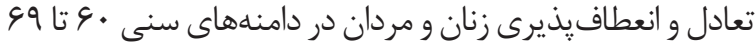

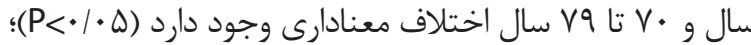

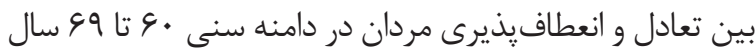

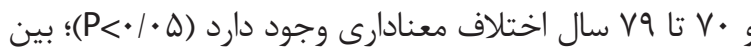

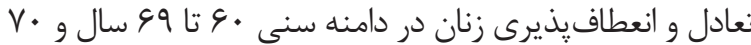

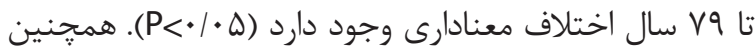

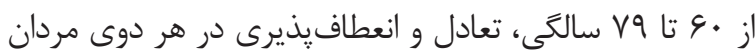

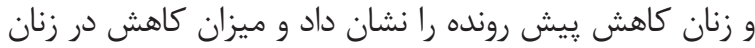

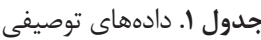

\begin{tabular}{|c|c|c|c|}
\hline مردان & زنان & دامنه سنى & متغيرها \\
\hline$r N / \Delta \cdot \pm r / \Lambda$. & $r \varepsilon / \cdot r \pm r / \Lambda$. & $9 \cdot-99$ & \multirow{2}{*}{ انعطافيذيرى (سانتىمتر) } \\
\hline$r r / q . \pm r / v$. & $r M / \kappa \cdot \pm \Delta / \varepsilon$. & $v+-v q$ & \\
\hline$r F / \varepsilon \cdot \pm F / \cdot \Lambda$ & $r \cdot / q . \pm r / \cdot q$ & $9 \cdot-99$ & \multirow{2}{*}{ تعادل (سانتى متر) } \\
\hline$r \nu / r \pm \Psi / v$. & $\mid V / \varepsilon \cdot \pm F / c$. & $v \cdot-v q$ & \\
\hline
\end{tabular}

L 
جدول r. نتايج آزمونهاى t وابسته و مستقل

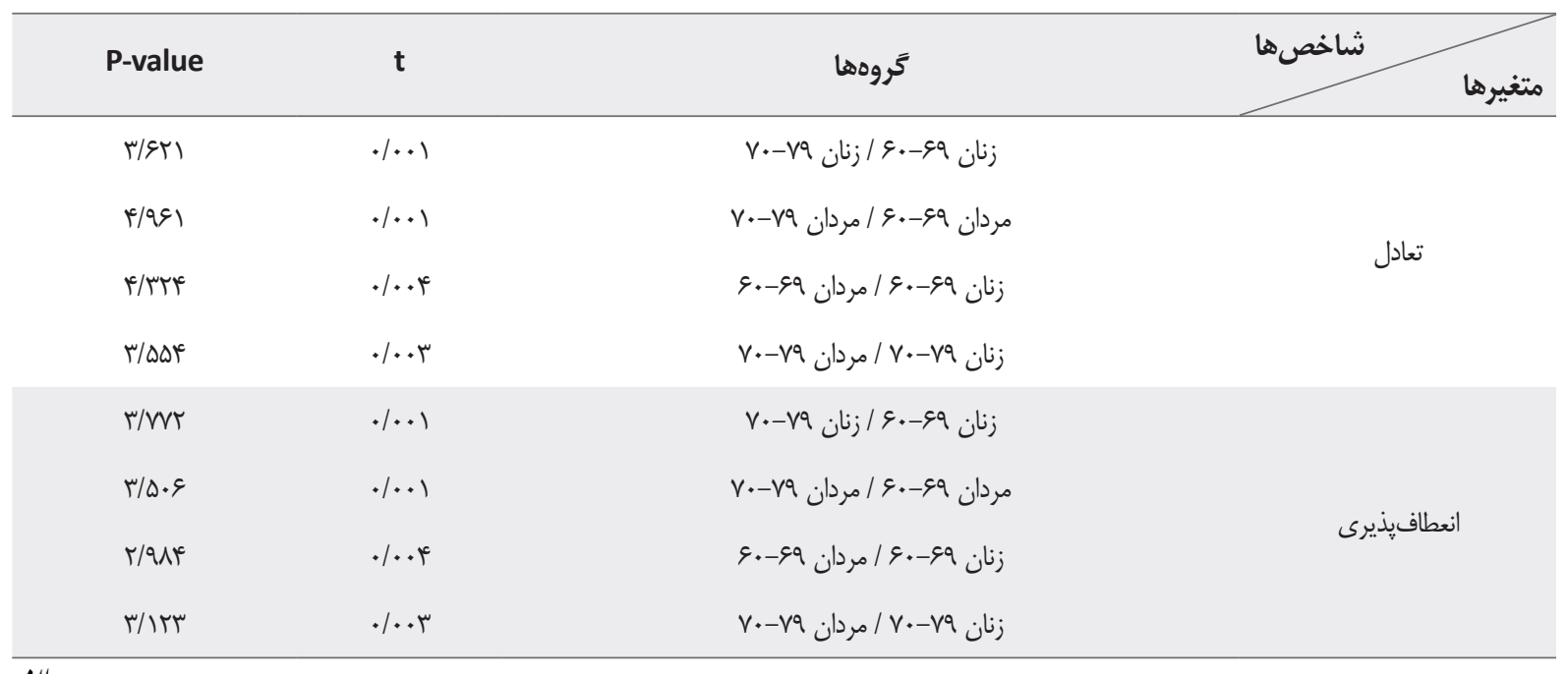

تعادل كاهش مىيابد و اين كاهش نيز در زنان بيشتر از مردان

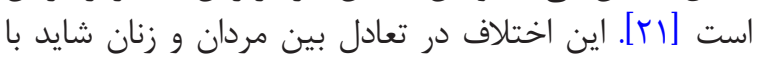

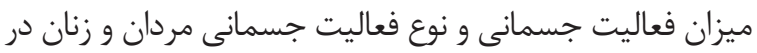

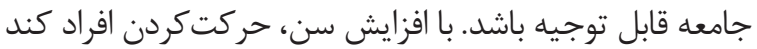

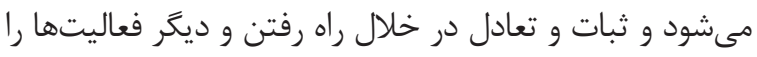

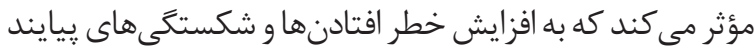

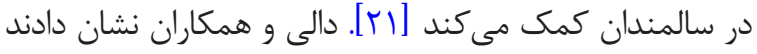

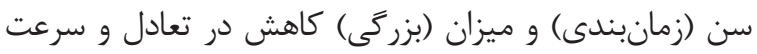

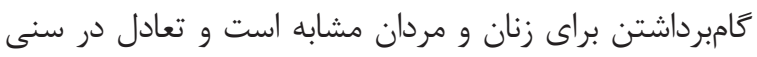

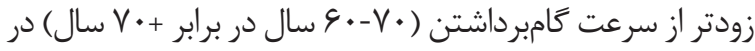

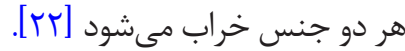

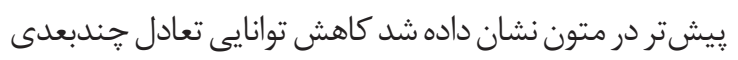

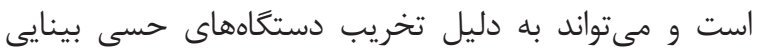

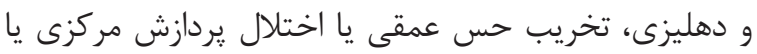

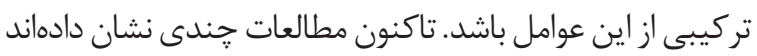

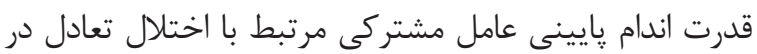

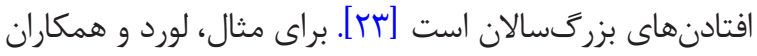

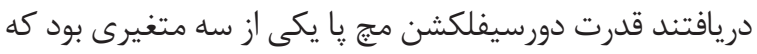

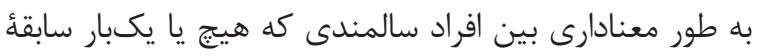

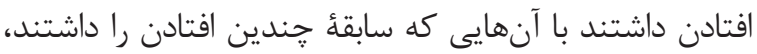
تمايز قائل شد [بr]

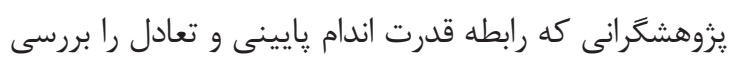

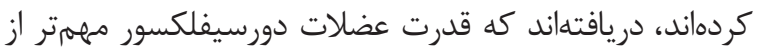

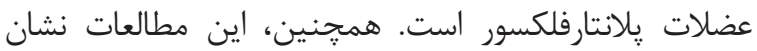
مىدهند رابطهاى قوى بين قدرت اندام پايينى و توانايى براى

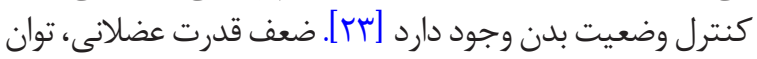
اندامهاى يايينى، تعادل و كنترل وضعيت بدن و وتوانايى راه راه رفتن،

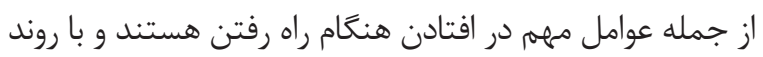

كاهش اجتنابنايذير در توده عضله و در نتيجه كاهش قدرت

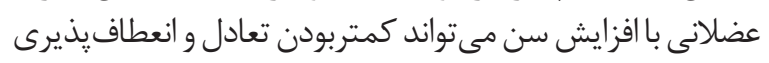

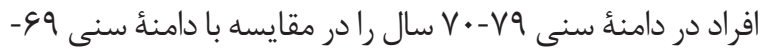

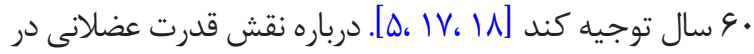

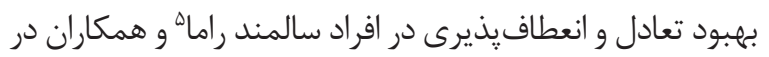

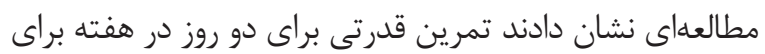

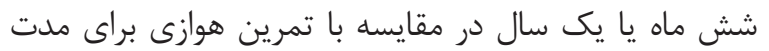

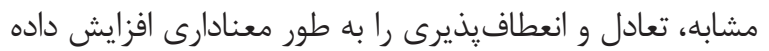

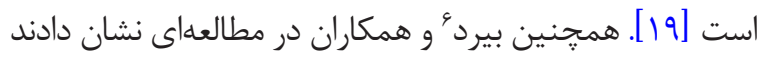

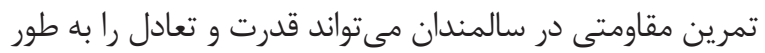

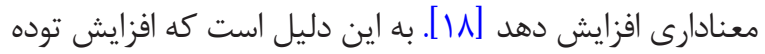
و قدرت عضله هدف مهرمى در يزشكى توانبخشى دهن، ساركوينيا و

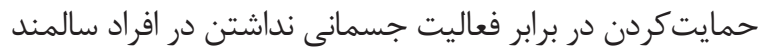
در نظر كرفته شده است [ب. تعادل مىتواند با دامنهُ وسيعى از بيمارىها، آسيبها يا

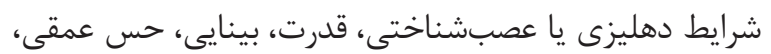

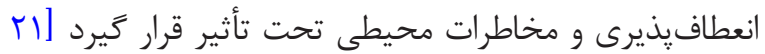

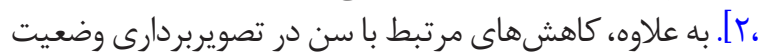

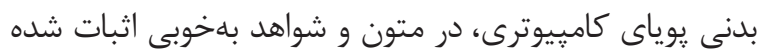

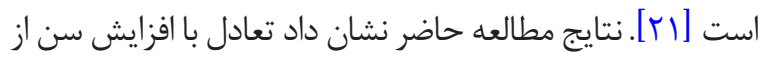

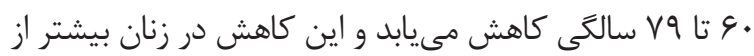

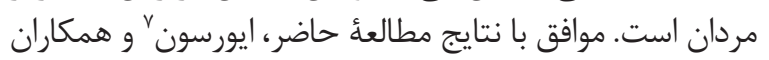

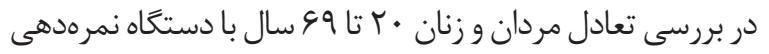

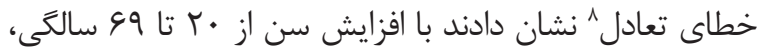

8. Balance Error Scoring System 
فعاليتهايى شامل خمشدن و دسترسى به اشياى مهم است

انعطافيذيرى خوب، سلامتى عضلات و مفاصل را ارتقا

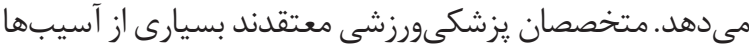

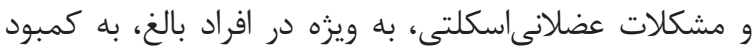

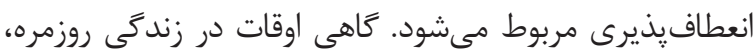

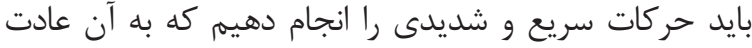

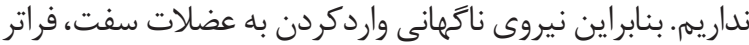

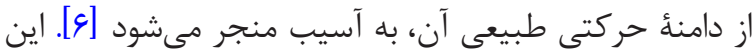

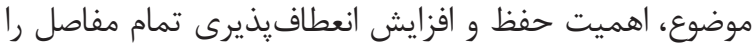

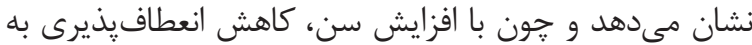

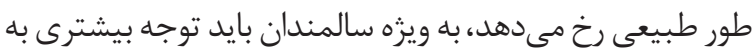

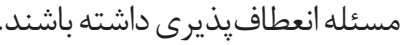

با توجه به تفاوتهاى جنسى، بيشترين مطالعات نشان

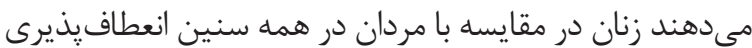

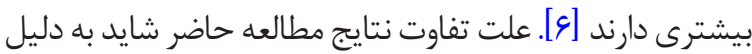

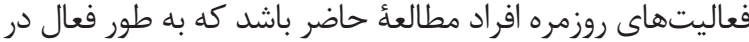

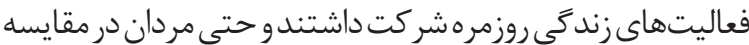

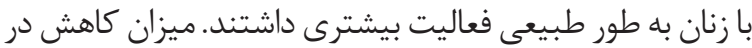

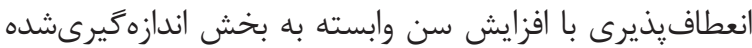

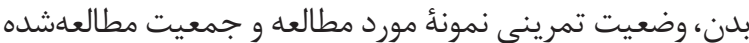

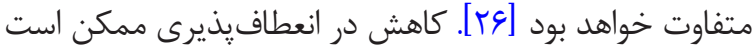

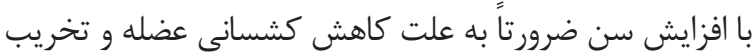

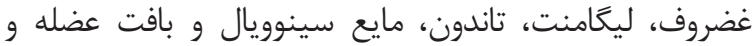

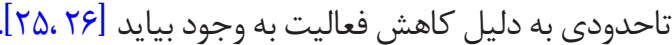

يروهشكران زيادى بهبودهاى معنادارى در تعدادى از عوامل

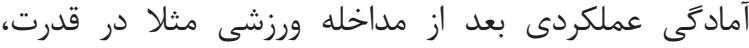

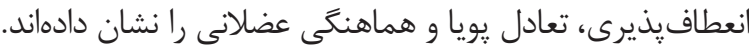

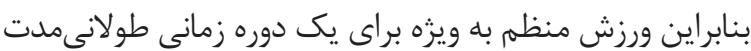

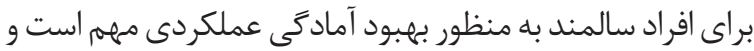

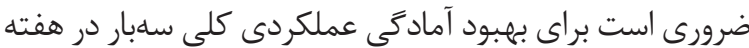

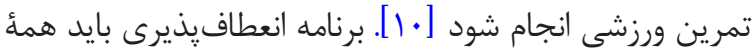

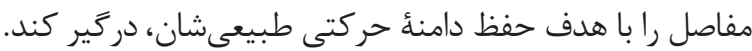

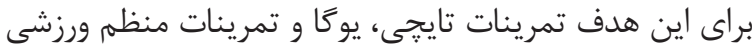

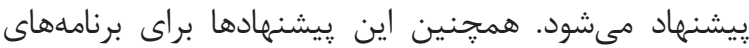

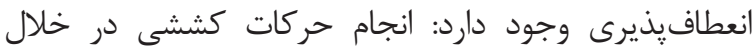

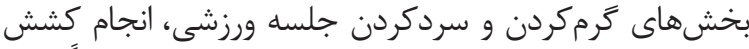

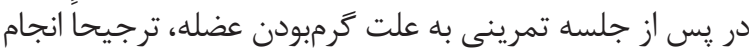

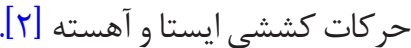
با توجه به محدوديتهاى حركتى و عملكردى افراد سالمند،

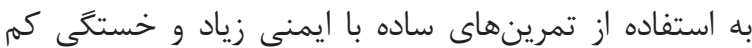

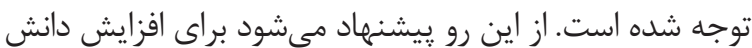

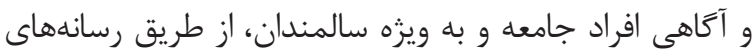

كهولت به طور ييشروندهاى ضعيف مىشوند [ع] . نتايج راجع به

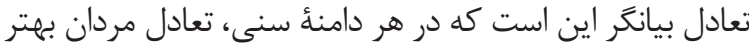

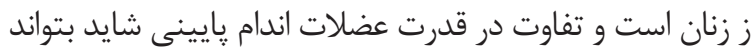

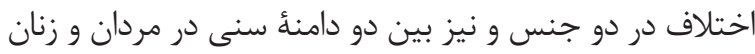

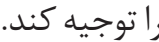

با توجه به اينكه تعادل معيوب با افزايش خطر افتادنها، ناتوانى

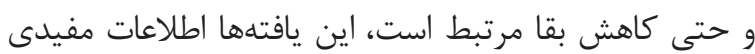

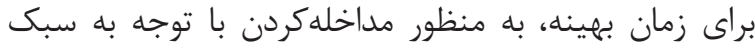

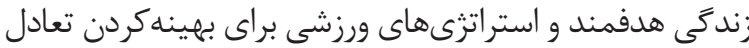

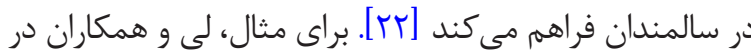

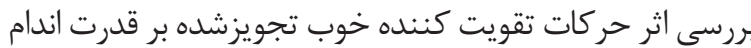

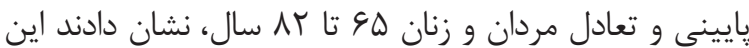

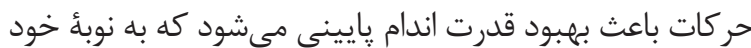

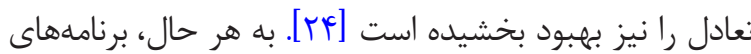

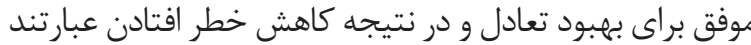

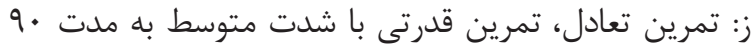

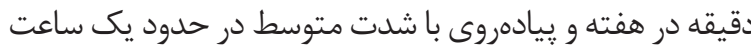

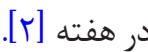

انعطافيذيرى جزوى ضرورى از توانايى انجام فعاليتهاى

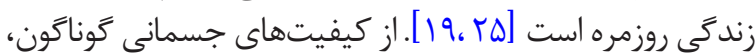

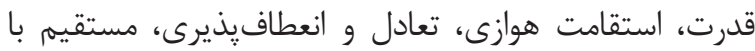

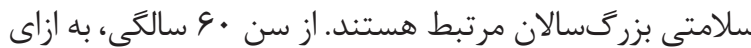

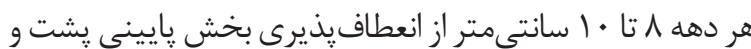

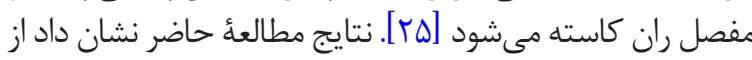

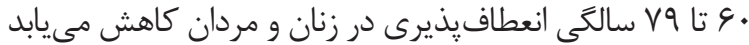

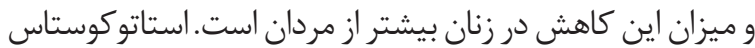

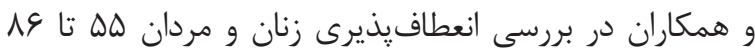

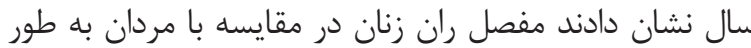

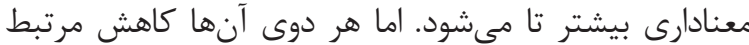

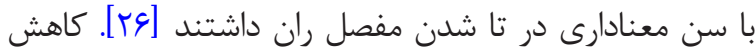

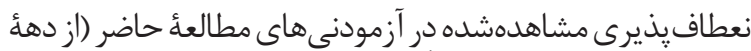

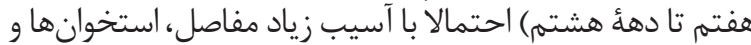

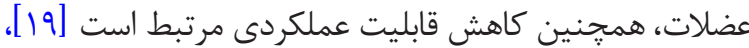

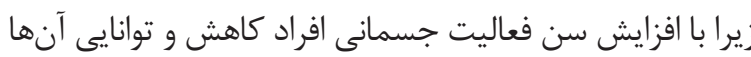

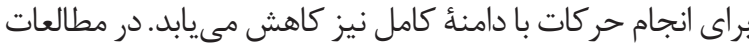

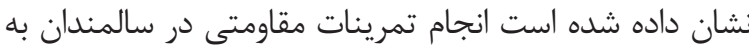

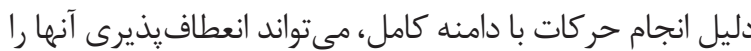

افزايش دهد [19] إن]

انعطافيذيرى مفصل ممكن است در طول عمر كاهش يابد؛

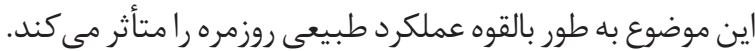

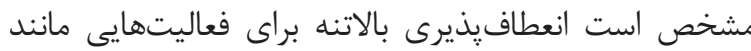

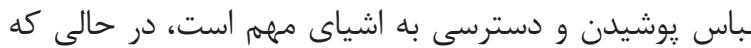

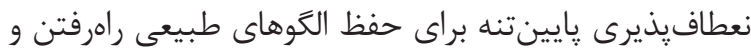


آزمونهاى انجام شده باشد كه البته با توجه به توانايى يزوهشگر

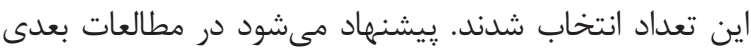

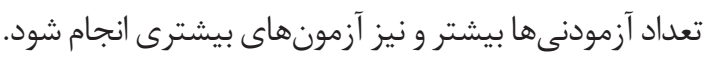

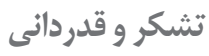

بدين وسيله از تمامى آزمودنىهاى مطالعه حاضر به دليل

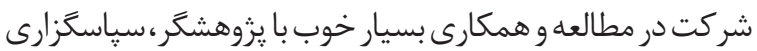

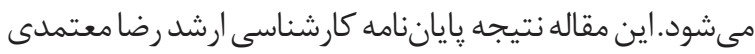
است كه با هزينه شخصى انجام شده است.
مختلف اقدامات جدى آمرى انجام گيرد و متخصصان ورزشى به

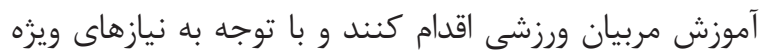

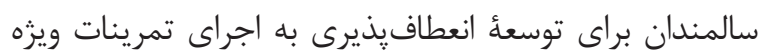

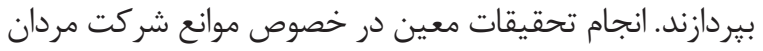

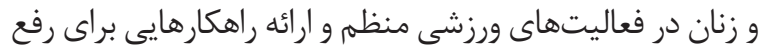

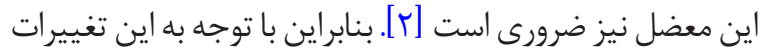

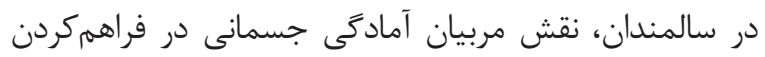
برنامههاى مناسب براى ارتقاو حفظ تندرستى افراد سالمني المند بسيار

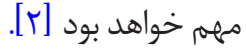

اهداف اصلى برنامههاى آمادگى براى افراد سالمند بايد براى

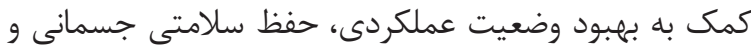

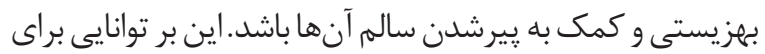

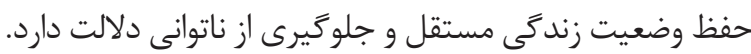

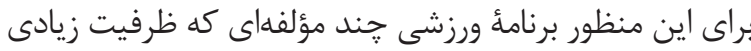

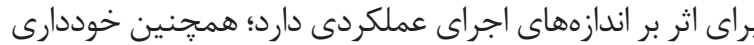

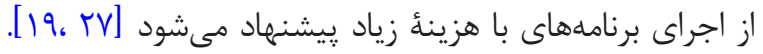

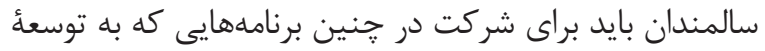

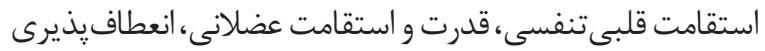

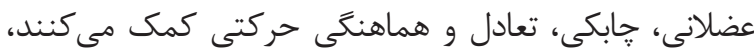

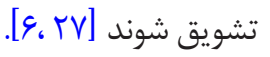

\section{نتيجه كيرى نهايى}

در راستاى نتايج مطالعهُ حاضر و با توجه به كاهش تعادل

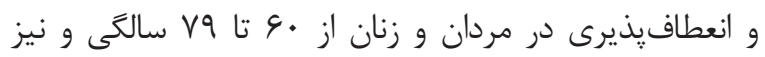

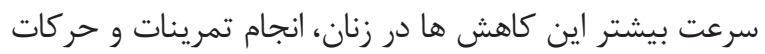

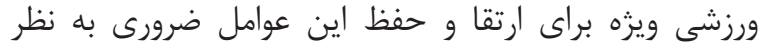

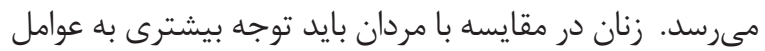

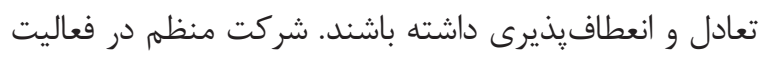

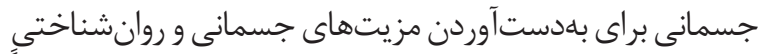

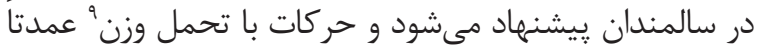

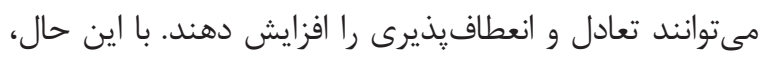

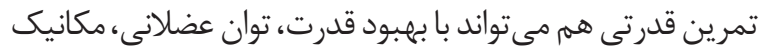

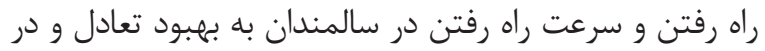

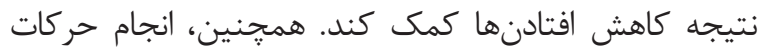

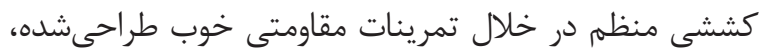

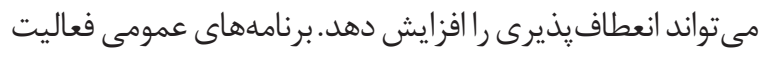

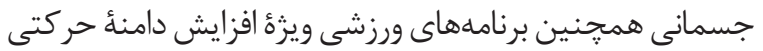

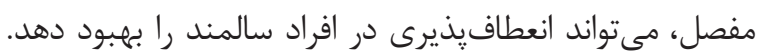

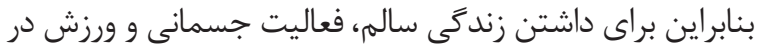

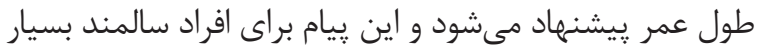
مبرم است.

از محدوديتهاى اين مطالعه شايد تعداد كم نمونهها و 


\section{References}

[1] Chehrehnegar N, Keshavarzi F, Rahnamaee N, Aghajafari Z. [Relationship between visual constructive abilities and activity of daily living in home dwelling elderly population (Persian)]. Iranian Journal of Ageing. 2016; 11(2):220-25.

[2] Howley ET, Thompson DL. Fitness Professional's Handbook. 6th edition. Champaign: Human Kinetics; 2012.

[3] Minasian V, Marandi SM, Mojtahedi H, Ghasemi Gh. [The evaluation of health-related physical fitness status of men aged between 50 and 65 in Isfahan and comparison with available norms (Persian)]. Journal of Sport Bioscience. 2012; 4(14):111-27. doi: 10.22059/JSB.2013.29531

[4] Gaeeni AA, Rajabi H. [Physical fitness (Persian)]. Tehran: Samt; 2004.

[5] Doherty TJ. Invited review: Aging and sarcopenia. Journal of Applied Physiology. 2003; 95(4):1717-27. [DOI:10.1152/japplt physiol.00347.2003]

[6] Hoeger WWK, Hoeger SA. Lifetime physical fitness \& wellness: A personalized program. Boston, Massachusetts: Cengage Learning; 2013.

[7] McArdle A, Vasilaki A, Jackson M. Exercise and skeletal muscle ageing: Cellular and molecular mechanisms. Ageing Research Reviews. 2002; 1(1):79-93. [DOI:10.1016/S0047-6374(01)00368-2]

[8] Peake J, Gatta PD, Cameron-Smith D. Aging and its effects on inflammation in skeletal muscle at rest and following exercise-induced muscle injury. American Journal of Physiology-Regulatory, Integrative and Comparative Physiology. 2010; 298(6):R1485R95. [DOI:10.1152/ajpregu.00467.2009]

[9] American College of Sports Medicine. Health-related physical fitness assessment manual. Philadelphia, Pennsylvania: Lippincott Williams \& Wilkins; 2014

[10] Nakamura Y, Tanaka K, Yabushita N, Sakai T, Shigematsu R. Effects of exercise frequency on functional fitness in older adult women. Archives of Gerontology and Geriatrics. 2007; 44(2):16373. [DOI:10.1016/j.archger.2006.04.007]

[11] Takeshima N, Rogers NL, Rogers ME, Islam Daisuke Koizumi MM, Lee S. Functional fitness gain varies in older adults depending on exercise mode. Medicine \& Science in Sports \& Exercise. 2007; 39(11):2036-43. [DOI:10.1249/mss.0b013e31814844b7]

[12] Hrysomallis C. Balance ability and athletic performance. Sports Medicine. 2011; 41(3):221-32. [DOI:10.2165/11538560-00000000000000]

[13] Shubert TE, Schrodt LA, Mercer VS, Busby-Whitehead J, Giuliani CA. Are Scores on Balance Screening Tests Associated with Mobility in Older Adults? Journal of Geriatric Physical Therapy. 2005; 29(1):33-9. [DOI:10.1519/00139143-200604000-00007]

[14] Duncan PW, Weiner DK, Chandler J, Studenski S. Functional reach: A new clinical measure of balance. Journal of Gerontology. 1990; 45(6):192-7. [DOI:10.1093/geronj/45.6.M192]

[15] Hurley BF, Roth SM. Strength training in the elderly: Effects on risk factors for age-related diseases. Sports Medicine. 2000; 30(4):249-68. [DOI:10.2165/00007256-200030040-00002]

[16] American College of Sports Medicine. Guidelines for exercise testing and prescription. Philadelphia, Pennsylvania: Lippincott Williams \& Wilkins; 2014.
[17] Raj IS, Bird SR, Shield AJ. Aging and the force-velocity relationship of muscles. Experimental Gerontology. 2010; 45(2):81-90. [DOI:10.1016/j.exger.2009.10.013]

[18] Seco J, Abecia LC, Echevarria E, Barbero I, Torres-Unda J, Rodriguez $\mathrm{V}$, et al. A long-term physical activity training program increases strength and flexibility, and improves balance in older adults. Rehabilitation Nursing. 2012; 38(1):37-47. [DOI:10.1002/ rnj.64]

[19] Roma MFB, Busse AL, Betoni RA, de Melo AC, Kong J, Santarem JM, et al. Effects of resistance training and aerobic exercise in elderly people concerning physical fitness and ability: a prospective clinical trial. Einstein. 2013; 11(2):153-7. [DOI:10.1590/ S1679-45082013000200003]

[20] Popov DV, Lysenko EA, Bachinin AV, Miller TF, Kurochkina NS, Kravchenko IV, et al. The influence of resistance exercise intensity and metabolic stress on anabolic signaling and the expression of myogenic genes in skeletal muscle. Muscle Nerve. 2015; 51(3):434-42. [DOI:10.1002/mus.24314]

[21] Iverson GL. Koehle MS. Normative data for the balance error scoring system in adults. Rehabilitation Research and Practice. 2013; Article ID:846418. [DOI:10.1155/2013/846418]

[22] Ryall JG, Schertzer JD, Lynch GS. Cellular and molecular mechanisms underlying age-related skeletal muscle wasting and weakness. Biogerontology. 2008; 9(4):213-28. [DOI:10.1007/ s10522-008-9131-0]

[23] Daly RM, Rosengren BE, Alwis G, Ahlborg HG, Sernbo I, Karlsson MK. Gender specific age-related changes in bone density, muscle strength and functional performance in the elderly: a-10 year prospective population-based study. BMC Geriatrics. 2013; 13(1):71. [DOI:10.1186/1471-2318-13-71]

[24] Hess JA, Woollacott M. Effect of high-intensity strength training on functional measures of balance ability in balance-impaired older adults. Journal of Manipulative and Physiological Therapeutics. 2005; 28(8):582-90. [DOI:10.1016/j.jmpt.2005.08.013]

[25] Lee IH, Park SY. Balance improvement by strength training for the elderly. Journal of Physical Therapy Science. 2013; 25(12):1591-3. [DOI:10.1589/jpts.25.1591]

[26] Stathokostas L, McDonald MW, Little RMD, Paterson DH Flexibility of older adults aged 55-86 years and the influence of physical activity. Journal of Aging Research. 2013; 1-8. [DOI:10.1155/2013/743843]

[27] Vi-a J, Salvador-Pascual A, Tarazona-Santabalbina FJ, Rodriguez-Ma-as L, Gomez-Cabrera MC. Exercise training as a drug to treat age associated frailty. Free Radical Biology and Medicine. 2016; 98:159-64. [DOI:10.1016/j.freeradbiomed.2016.03.024] 
